Heart. 2014 October ; 100(20): 1569-1570. doi:10.1136/heartjnl-2014-306111.

\title{
Genomics in clinical practice
}

\author{
James R Priest ${ }^{1,2}$ and Euan A Ashley ${ }^{2,3}$ \\ ${ }^{1}$ Division of Pediatric Cardiology, Stanford University School of Medicine, Stanford University, \\ Stanford, California, USA \\ ${ }^{2}$ Stanford Cardiovascular Institute, Stanford University School of Medicine, Stanford, California, \\ USA \\ ${ }^{3}$ Division of Cardiovascular Medicine, Stanford University School of Medicine, Stanford University, \\ Stanford, California, USA
}

Prior to the completion of the human genome project and establishment of the phrase "personalized medicine", physicians and scientists forecasted the transformative impact of human genetics upon the understanding and treatment of human disease. Within the field of cardiovascular medicine, the current application of clinical genetic testing is generally limited to inherited arrhythmias, cardiomyopathies, occasional pharmacogenomic profiling for anticoagulation and antiplatelet therapies, and rarely to establish syndromic diagnoses. Even among informed practitioners on the wards and in the clinics, the skeptics among us observe that the rapid proliferation of genome wide association studies (GWAS) which characterize the contribution of common genetic variation to common diseases contrasts sharply with their infrequent application to everyday clinical practice. Some cardiologists may argue that advances in genetic basis of cardiovascular disease do not inform us any more than a family history. ${ }^{1}$ The authors of the study published in this issue of Heart have endeavored to bridge the divide between our growing knowledge of cardiovascular genomics and the application of genetics to routine clinical practice. ${ }^{2}$

In a cohort of 460 individuals for whom the first presentation of coronary artery disease was acute coronary syndrome, Labos et al. demonstrated that a combined genetic risk score (GRS) incorporating 30 validated SNPs had moderate predictive value in a clinical model adjusted for traditional risk factors. The predictive value of the GRS was exceeded by hypertension, smoking, and BMI, but remarkably the association between GRS and earlier incident disease was more statistically significant than diabetes and hypercholesterolemia with a clinically meaningful effect size. The GRS included variants within genes such as LPA, APOA5, LDLR, and PCSK 9 which drive traditional risk factors such as lipid levels and composition, however the GRS also included genes such as TCF 21 which plays a role in endothelial biology. ${ }^{3}$ The role of coronary artery endothelium and smooth muscle cells within the pathophysiology of plaque formation is demonstrated in the research setting, but

Correspondence to Dr Euan A Ashley, Division of Cardiovascular Medicine, Stanford University School of Medicine, 300 Pasteur Drive, A265 MC 5319, Stanford, CA 94305, USA; euan@stanford.edu.

Competing interests EAA is a founder of Personalis Inc., which offers clinical genetic testing.

Provenance and peer review Commissioned; internally peer reviewed. 
is not measured directly by the risk factors employed in daily clinical practice. Thus by combining a group of genetic variants playing different roles in the pathophysiology of coronary artery disease, the authors captured new and important clinical information, which adds independent predictive power to population modeling of clinical risk for age of incident coronary artery disease.

Adding genetic markers to clinically actionable risk factors for coronary artery disease such as lipid metabolism, thrombotic risk, and inflammation is the clear point of entry for harnessing genetics to improve the care of patients with coronary artery disease. The common genetic contributors for these diseases are well validated, the technology for genotyping is readily available in the clinic, and as the authors have shown demonstrably useful to the clinician and patient. Were this type of genetic information a novel blood test or diagnostic procedure, the calls for a gold-standard randomized controlled clinical trial would have been answered years ago. Indeed, studies such as this one, which add specific genetic information to answer a focused clinical question are long overdue, particularly in the field of adult cardiovascular medicine which has been at the vanguard of assembling high quality clinical evidence and applying it equitably to improve the health of large patient populations. We look forward to the outcomes of randomized controlled trials which leverage genetic information to target drug therapies and improve the care of patients with cardiovascular disease. ${ }^{4}$ The full and direct public benefit of the genetic information learned from GWAS will not be realized until prospective trials are completed and their findings integrated into daily clinical practice. In contrast to a new pharmaceutical compound or device, there is little financial incentive to conduct targeted clinical trials for a relatively inexpensive genetic test. Thus, it seems the clinical trials necessary to integrate and implement genetic testing into daily clinical practice will almost certainly require additional sustained public funding.

The technologies for detecting genetic variation have advanced at a rapid pace in the last decade allowing the delineation of most of the genetic variation within a single individual, including both the common genetic variation described in GWAS and rare familial and private variation. Many laboratories around the world have applied these next-generation sequencing tools to the comprehensive analysis of patients in the clinic and the intensive care unit. ${ }^{56}$ This work has illustrated clearly the need for integrated models of care, which bring genetics into the daily practice of each clinical department. The integrated model of care includes two important types of practitioner, and each clinical department requires the full participation of both physician champions and genetic counselors each with specialized knowledge of the interaction between their clinical discipline and the relevant genetic risk factors. The prototype for an integrated model of genetic care can already be found in a handful of subspecialty clinics such as high-risk obstetrics and oncology where physicians and subspecialty genetic counselors work closely together.

Finally we know what genomics is today, but know not what it may be in the future. The wealth of useful data produced by the field of genomics leads us to consider other types of comprehensive "omics" datasets such as transcriptomics, proteomics, and metabolomics which show extraordinary potential for informing the diagnosis and treatment of human disease. ${ }^{7}$ As more studies demonstrate the clinical utility of "omics" datasets, it is incumbent upon each medical specialty to ask carefully-defined questions to delineate the clinical 
scenarios where a particular type of clinical genomics data may improve patient care such as by reducing the number of invasive biopsies following heart transplant. ${ }^{8}$ The accompanying study by Labos et al. exemplifies the focused preliminary work that will be required to integrate genomic data into the clinical care of patients at risk for coronary artery disease.

\section{References}

1. Paynter NP, Chasman DI, Paré G, Buring JE, Cook NR, Miletich JP, Ridker PM. Association Between a Literature-Based Genetic Risk Score and Cardiovascular Events in Women. JAMA. 2010; 303:631-7. [PubMed: 20159871]

2. Labos C, Wang RHL, Pilote L, et al. Traditional Risk Factors and a Genetic Risk Score Are Associated with Age of First Acute Coronary Syndrome. Heart. 2014; 100:1620-4. [PubMed: 24842871]

3. Miller CL, Anderson DR, Kundu RK, et al. Disease-related growth factor and embryonic signaling pathways modulate an enhancer of TCF21 expression at the 6q23.2 coronary heart disease locus. PLoS Genet. 2013; 9(7)

4. Ioannidis JP. Genetic Prediction for Common Diseases. Archives of internal medicine. 2012; 172:744-6. [PubMed: 22782208]

5. Dewey FE, Grove ME, Pan C, et al. Clinical interpretation and implications of whole-genome sequencing. JAMA. 2014; 311:1035-45. JAMA 2014; 311:1035-45. [PubMed: 24618965]

6. Saunders CJ, Miller NA, Soden SE, et al. Rapid whole-genome sequencing for genetic disease diagnosis in neonatal intensive care units. Sci Transl Med. 2012; 4:154.

7. Chen R, Mias GI, Li-Pook-Than J, et al. Personal omics profiling reveals dynamic molecular and medical phenotypes. Cell. 2012; 148:1293-1307. [PubMed: 22424236]

8. De Vlaminck I, Valentine HA, Snyder TM, et al. Circulating Cell-Free DNA Enables Noninvasive Diagnosis of Heart Transplant Rejection. Sci Transl Med. 2014:241ra77. 